LETTERS TO THE EDITOR

\title{
Antibodies to NMDA receptor, blood-brain barrier disruption and schizophrenia: a theory with unproven links
}

Molecular Psychiatry (2014) 19, 1054; doi:10.1038/mp.2014.25; published online 25 March 2014

Hammer et al. ${ }^{1}$ examined 2817 subjects, including 1325 healthy subjects and patients with schizophrenia (1081), Parkinson disease (263) and affective disorders (148) for the presence of several immunoglobulin subtypes of antibodies against the NR1 subunit of the NMDA receptor (NMDAR). In this large-scale screening, they found that $10.5 \%$ of all subjects were seropositive. The authors conclude that schizophrenic individuals with a past or present history of blood-brain barrier (BBB) disturbance are more likely to have more pronounced neurological symptoms upon NMDAR antibody seropositivity. However, there is no data supporting this conclusion in any of the sections of the manuscript:

(1) The authors' conclusion was based on retrospective review of clinical information for possible neurotrauma at birth or thereafter in the patients and controls. Even assuming that there was neurotrauma (which in fact is not clearly demonstrated), the authors do not provide any evidence that it altered the integrity of the BBB.

(2) In the experiments with cultured neurons the authors claim that decreased NMDAR immunostaining was visible with confocal microscopy after incubation with patients' antibodies for $20 \mathrm{~min}$, 'subsequently placing the cells in pre-warmed growth medium for 15 min to allow internalization.' However, other than a graph, there is no data showing confocal microscopy pictures of the density of cell surface/synaptic NMDAR clusters before and after incubation with patients' antibodies. The findings are hard to explain given that studies by other investigators on antibody-mediated NMDAR internalization using patient samples with very high titer antibodies (for example, those with anti-NMDAR encephalitis) found no detectable internalization using confocal microscopy until $\sim 4 \mathrm{~h}$ after neuronal incubation, reaching a plateau at $\sim 24 \mathrm{~h}^{2,3}$

(3) In contrast to the authors' suggestion, the mouse experiments do not provide supporting evidence to their interpretation of the patients' findings (point 1). For this, in the minimum, the authors should demonstrate that the systemic injection of patients' immunoglobulin $\mathrm{G}$ ( $\mathrm{lgG}$ ) reaches the brain of mice, binds to NMDAR and alters receptor levels and function.

In a clinical series by Steiner et al.o ${ }^{4}$ including 459 subjects (121 with schizophrenia, 70 major depression, 38 borderline personality disorder (BLPD) and 230 controls), none had lgG NR1 subtype antibodies (two positive cases had anti-NMDAR encephalitis) - a finding closely resembling the data of Hammer et al. ${ }^{1} \quad(18 / 2817,13$ patients, 5 controls; 0.6\%). However, Steiner et al. ${ }^{4}$ found that $9.9 \%$ patients with schizophrenia, 2.8\% major depression, 0\% BLPD and 0.4\% controls had IgA or IgM antibodies, or IgG antibodies against NR1/NR2. The different incidence of NMDAR antibodies in healthy subjects of both series $(137 / 1325(10.8 \%)$ versus $1 / 230(0.4 \%))$ is surprising.

Overall, these findings cast doubt on the interpretation that Hammer et al. ${ }^{1}$ made on their results, and show the difficulty that these authors and Steiner et $a l^{4}{ }^{4}$ encountered in the interpretation of antibody testing (using only serum and a cell-based assay), a problem we recently reported..$^{5}$ In our opinion, the study of Hammer et al. ${ }^{1}$ merely shows that NR1 lgG antibodies are extremely uncommon in patients with schizophrenia, and $\lg \mathrm{A}$ or $\lg \mathrm{M}$ antibodies are frequent among healthy individuals, lacking specificity.

\section{CONFLICT OF INTEREST}

Dr Dalmau holds a patent application for the use of NMDA receptor as antibody test and has received a research grant for Euroimmun. Dr Titulaer received a travel grant for lecturing in India from Sun Pharma, India.

\section{ACKNOWLEDGMENTS}

Dr Titulaer is supported by grants from the Netherlands Organisation for Scientific Research (NWO, Veni-incentive), the Dutch Epilepsy Foundations (project number 14-19), an ErasmusMC fellowship and previously a clinical research fellowship by the Dutch Cancer Society (2009-4451). Dr Dalmau is supported by grants from the National Institutes of Health RO1NS077851, Fundació la Marató TV3 and Fondo de Investigaciones Sanitarias (FIS, PI11/01780).

$$
\begin{array}{r}
\text { MJ Titulaer }{ }^{1,2} \text { and J Dalmau }{ }^{1,3} \\
\text { 'Department of Neurology at Hospital Clinic-Institut } \\
d^{\prime} \text { Investigacions Biomèdiques August Pi i Sunyer (IDIBAPS), } \\
\text { Barcelona, Spain; } \\
{ }^{2} \text { Erasmus Medical Center, Department of Neurology, Rotterdam, } \\
\text { The Netherlands and } \\
{ }^{3} \text { Institució Catalana de Recerca i Estudis Avançats (ICREA), } \\
\text { Barcelona, Spain } \\
\text { E-mail: Josep.dalmau@uphs.upenn.edu }
\end{array}
$$

\section{REFERENCES}

1 Hammer C, Stepniak B, Schneider A, Papiol S, Tantra M, Begemann M et al. Mol Psychiatry 2014; 19: 1143-1149.

2 Hughes EG, Peng X, Gleichman AJ, Lai M, Zhou L, Tsou R et al. J Neurosci 2010; 30 5866-5875.

3 Moscato E, Peng XY, Parsons T, Dalmau J, Balice-Gordon R. J Neuroimmunol 2012; 253: 145 (abstract 408).

4 Steiner J, Walter M, Glanz W, Sarnyai Z, Bernstein HG, Vielhaber S et al. JAMA Psychiatry 2013; 70: 271-278.

5 Gresa-Arribas N, Titulaer MJ, Torrents A, Aguilar E, McCracken L, Leypoldt F et al. Lancet Neurol 2013; 13: 167-177.

\section{Apolipoprotein E4 carrier status plus circulating anti-NMDAR 1 autoantibodies: association with schizoaffective disorder}

Molecular Psychiatry (2014) 19, 1054-1056; doi:10.1038/mp.2014.52; published online 3 June 2014

In a Letter to the Editor, Titulaer and Dalmau ${ }^{1}$ commented on our recent publication, ${ }^{2}$ calling for a direct response for scientific 
Table 1. Combined presence of APOE4 carrier status and NMDAR-AB in psychiatric patients

\begin{tabular}{|c|c|c|c|}
\hline Study group & $\begin{array}{l}\text { No combination of ApoE4 carrier status and } \\
\qquad N M D A R-A B, N(\%)\end{array}$ & $\begin{array}{c}\text { ApoE4 carrier+NMDAR-AB, } \\
N(\%)\end{array}$ & $\begin{array}{c}P(A p o E 4 \times N M D A R-A B) \\
(O R(C I 95 \%))\end{array}$ \\
\hline $\begin{array}{l}\text { Schizophrenia patients, GRAS } \\
(N=875)\end{array}$ & $852(97.4)$ & $23(2.6)$ & $0.163(1.616(0.824-3.170))$ \\
\hline $\begin{array}{l}\text { Schizoaffective patients, GRAS } \\
(N=200)\end{array}$ & $189(94.5)$ & $11(5.5)$ & $0.001(6.109(2.027-18.415))$ \\
\hline Bipolar patients $(N=60)$ & $56(93.3)$ & $4(6.7)$ & $0.223(2.562(0.564-11.632))$ \\
\hline Unipolar depressed patients $(N=88)$ & $84(95.5)$ & $4(4.5)$ & $0.563(1.497(0.382-5.861))$ \\
\hline Healthy GRAS controls $(N=1269)$ & $1240(97.7)$ & $29(2.3)$ & \\
\hline Total $(N=2492)$ & $2421(97.2)$ & $71(2.8)$ & \\
\hline
\end{tabular}

Abbreviations: $\mathrm{Cl}$, confidence intervals; GRAS, Göttingen Research Association for Schizophrenia-Data Collection; OR, relative odds. $P$-values are displayed for the interaction of ApoE4 carrier status and NMDAR-AB status in binary logistic regression. NMDAR-AB status, ApoE4 carrier status and age served as covariates. Significant $P$-values are displayed in bold.

accuracy. We reported that autoantibodies against the NR1 subunit of the $N$-methyl-D-aspartate receptor (NMDAR-AB) are highly seroprevalent in healthy and in neuropsychiatrically ill subjects. ${ }^{2}$ This unexpected finding has now been fully reproduced by Steiner et $a l^{3}$ who provided an addendum to their previous paper, cited by Titulaer and Dalmau, ${ }^{1}$ revising their earlier results on healthy individuals. ${ }^{4}$ Any further comments on this topic are thus outdated.

More importantly, Titulaer and Dalmau ${ }^{1}$ question the validity of a widely used cell biological technique for measuring the internalization of membrane proteins from the cell surface. In fact, endocytosis duration determines the endocytic compartment, with early endosome appearing after 5-10 min, already recycling endosomes at $30 \mathrm{~min}$, and late endosomes at 30-60 min. Thus, after $\geqslant 30$ min one would expect recycling to occur. ${ }^{5,6}$ Titulaer and Dalmau ${ }^{1}$ cite a paper that does not investigate internalization. ${ }^{7}$ It shows in cultured neurons consequences on surface NMDAR1 expression of an exposure for 1-7 days to human anti-NMDAR1 immunoglobulin $(\mathrm{lg}) \mathrm{G}$. Although this time frame is certainly long enough to include endocytosis, days of serum contact allow for many secondary adaptations, including well-known toxicity effects that have not been distinguished by the authors from the internalization of NR1. In contrast, we did analyze endocytosis and found exactly what was predicted, notably for all investigated Ig subclasses, IgG, IgA and $\lg \mathrm{M}$. We are adding representative confocal images, before and after permeabilisation of neurons, reflecting our experimental conditions (Supplementary Figure S1).

We also provided experimental evidence that circulating NMDAR-AB induced behavioral abnormalities only in $\mathrm{ApoE}^{-1-}$ mice with their known leaky blood-brain barrier (BBB), ${ }^{8}$ but not in respective controls. It was our specific intention to investigate behavior in this mouse model as a translational readout of psychiatric symptoms. Moreover, in a retrospective, hypothesisdriven analysis, we found more pronounced neurological symptoms in well-characterized NMDAR-AB seropositive patients with likely (but not proven) compromised BBB due to birth complications or past neurotrauma, ${ }^{2}$ in line with earlier studies (for review see ref. 9). To further support the hypothesis of a permeable BBB as prerequisite for circulating NMDAR-AB to influence brain function, we now provide additional data.

Here, we determined the apolipoprotein $E(A P O E)$ carrier status in a total of 2492 individuals tested twice independently for serum NMDAR-AB. ${ }^{2,10}$ DNA was isolated from blood using the JETQUICK Blood and Cell Culture Kit (Genomed, Loehe, Germany). The two single-nucleotide polymorphisms (SNPs) rs7412 and rs429358 were genotyped using the KASP genotyping system (LGC Genomics, Berlin, Germany). These two SNPs allow determination of the $A P O E$ allele status (APOE2, APOE3 and APOE4). Failed samples (3.7\%) were Sanger-sequenced using primers flanking both SNPs (forward: 5'-AACAACTGACCCCGGTGGCG-3', reverse:
5'-TCCGGCTGCCCATCTCCTCC-3'). Group differences in categorical variables were assessed using binary logistic regression, including NMDAR-AB and APOE4 carrier status, and age as covariates. Pearson's $X^{2} P$-values are displayed in Table 1 . In case of $N<5$ in one cell, Fisher's exact test was employed.

APOE4 carrier frequency was not significantly different in neuropsychiatric disease groups compared with controls (24.3\%) (Supplementary Table S1). The higher frequency in bipolar patients $(33.3 \%)$ did not reach statistical significance. NMDAR-AB were present in 71 individuals with at least one APOE4 allele $(2.8 \%$ of 2492 subjects). Whereas $X^{2}$ statistics did not show significant deviations from the theoretical distribution in case of schizophrenia, bipolar disorder, unipolar depressive patients and health, an excess of schizoaffective patients carried both APOE4 and NMDAR-AB $(P=0.001$, relative odds $(O R)=4.929)$ (Supplementary Table S2). Compared with controls, the interaction of APOE4 carrier status and NMDAR-AB seropositivity was significantly associated with schizoaffective disorder $(P=0.001, O R=6.109)$, corrected for APOE4 and NMDAR-AB main effects, and age (Table 1). Although the percentage of bipolar patients carrying both NMDAR-AB and APOE4 exceeded that of schizoaffective patients (6.7 vs $5.5 \%$ ), there was no significant association, possibly owing to low statistical power $(N=60)$. No difference was found with respect to disease phenotypes, or age at prodrome or disease onset after correction for multiple testing (Supplementary Tables S3a and b).

In summary, these novel results again highlight the importance of the BBB for NMDAR-AB-mediated pathology. APOE4 is associated with $\mathrm{BBB}$ leakage in mouse and man.,11 Delusions of grandiosity and mania are common psychiatric symptoms in anti-NMDAR encephalitis. ${ }^{12}$ Thus, NMDAR-AB may cause or boost these symptoms in neuropsychiatrically ill APOE4 carriers, which are then more likely diagnosed schizoaffective. Independent replication is necessary, but future studies investigating NMDAR-AB in neuropsychiatric disorders should consider factors determining BBB integrity.

\section{CONFLICT OF INTEREST}

The authors declare no conflict of interest.

C Hammer $^{1,2}$, M Zerche ${ }^{1}$, A Schneider ${ }^{2,3}$, M Begemann ${ }^{1,2}$, K-A Nave ${ }^{2,4}$ and H Ehrenreich ${ }^{1,2}$

${ }^{1}$ Clinical Neuroscience, Max Planck Institute of Experimental Medicine, Göttingen, Germany; ${ }^{2}$ DFG Center for Nanoscale Microscopy and Molecular Physiology of the Brain (CNMPB), Göttingen, Germany; ${ }^{3}$ German Center for Neurodegenerative Diseases (DZNE), Göttingen, Germany and

${ }^{4}$ Department of Neurogenetics, Max Planck Institute of Experimental Medicine, Göttingen, Germany Email: ehrenreich@em.mpg.de 


\section{REFERENCES}

1 Titulaer MJ, Dalmau J. Mol Psychiatry 2014; 19: 1054

2 Hammer C, Stepniak B, Schneider A, Papiol S, Tantra M, Begemann M et al. Mol Psychiatry 2014; 19: 1143-1149.

3 Steiner J, Walter M, Glanz W, Sarnyai Z, Bernstein HG, Vielhaber S et al. JAMA Psychiatry 2013; 70: 271-278.

4 Steiner J, Teegen B, Schiltz K, Bernstein HG, Stoecker W, Bogerts B. JAMA Psychiatry 2014 (in press)

5 de Juan-Sanz J, Zafra F, Lopez-Corcuera B, Aragon C. Traffic 2011; 12: 1850-1867.

6 Schneider A, Rajendran L, Honsho M, Gralle M, Donnert G, Wouters F et al. J Neurosci 2008; 28: 2874-2882.

7 Hughes EG, Peng X, Gleichman AJ, Lai M, Zhou L, Tsou R et al. J Neurosci 2010; 30 : 5866-5875

8 Bell RD, Winkler EA, Singh I, Sagare AP, Deane R, Wu Z et al. Nature 2012; 485: 512-516.

9 Shlosberg D, Benifla M, Kaufer D, Friedman A. Nat Rev Neurol 2010; 6: 393-403.

10 Dahm L, Ott C, Steiner J, Stepniak B, Teegen B, Saschenbrecker S et al. Ann Neurol 2014 (in press)

11 Halliday MR, Pomara N, Sagare AP, Mack WJ, Frangione B, Zlokovic BV. JAMA Neurol 2013; 70: 1198-1200.

12 Dalmau J, Lancaster E, Martinez-Hernandez E, Rosenfeld MR, Balice-Gordon R. Lancet Neurol 2011; 10: 63-74.

\section{cc) (9)}

This work is licensed under a Creative Commons AttributionNonCommercial-NoDerivs 3.0 Unported License. The images or other third party material in this article are included in the article's Creative Commons license, unless indicated otherwise in the credit line; if the material is not included under the Creative Commons license, users will need to obtain permission from the license holder to reproduce the material. To view a copy of this license, visit http://creativecommons.org/licenses/by-nc-nd/3.0/

Supplementary Information accompanies the paper on the Molecular Psychiatry website (http://www.nature.com/mp)

\section{No association between $R O R A$ polymorphisms and PTSD in two independent} samples

Molecular Psychiatry (2014) 19, 1056-1057; doi:10.1038/mp.2014.19; published online 22 July 2014

Logue et al. ${ }^{1}$ reported genome-wide significant association between a polymorphism ( $r$ s8042149) in the RORA gene, encoding the retinoic acid orphan receptor $A$, and posttraumatic stress disorder (PTSD) in a cohort of trauma-exposed white non-Hispanic US veterans and their partners. The genome-wide association study yielded evidence of association for three additional SNPs at the $10^{-6}$ threshold in the same cohort (rs8041061, rs8024133, rs11071561). Amstadter et al. $^{2}$ reported a significant association between rs8042149 and PTSD symptoms in the 2004 Florida Hurricane Study. The RORA gene encodes a nuclear hormone receptor that regulates the transcription activity of nearby genes. It is widely expressed in the brain, where it protects cortical neurons against oxidative stress-induced apoptosis by increasing the expression of antioxidant proteins. ${ }^{1}$ Logue et al. ${ }^{1}$ proposed that genetic variations in RORA may alter its expression, reducing the capacity of neurons to respond to biochemical stressors induced by traumatic stress.

We set out to replicate the association of variations in the RORA gene with lifetime risk of PTSD and to test whether RORA genotypes modified the association between cumulative trauma exposure and PTSD in two independent data sets.

The first data set was primarily European American-a PTSD subsample ( $N=2616$ trauma-exposed women) from the Nurses Health Study II (NHSII). ${ }^{3}$ The ascertainment of this data set and assessment of PTSD have been described. ${ }^{4}$ PTSD diagnosis was defined according to DSM-IV criteria ( $n=849$ cases, $n=1767$ traumaexposed controls). PTSD symptom severity was defined as the sum of the symptom ratings (1: 'not at all' to '5: extremely') across the 17 questions (mean $=32.73$, s.d. $=13.08$ ). PTSD symptom count was defined as the number of PTSD symptoms endorsed (mean $=7.15$, $\mathrm{s}$. $\mathrm{d} .=4.57$ ). A measure of cumulative trauma exposure was created by summing the number of different traumatic event types experienced. Four polymorphisms mapping to RORA were genotyped for each sample using the ABI PRISM 7900HT Sequence Detection System (Applied Biosystems, Foster City, CA, USA), in 384-well format or using the TaqMan OpenArrayTM SNP Genotyping Platform (Applied Biosystems) according to the manufacturer's instructions. All markers were in Hardy-Weinberg equilibrium. To reduce concern about population stratification, we restricted analysis to 2616 self-reported European-American women. Analyses were conducted in PLINK. ${ }^{5}$

SNPs rs8042149, the original genome-wide significant marker, rs11071561, rs8041061 and rs8024133 were not associated with lifetime PTSD under the additive, recessive or dominant models (Table 1).

SNPs rs8041061, rs8042149 and rs8024133 showed no evidence of association with either symptom severity or symptom count $(P$ values ranged from 0.06 to 0.54$)$. SNP rs 11071561 was associated with PTSD symptom severity $(P=0.02)$ in the same direction as in Logue et al., ${ }^{1}$ but was not associated with symptom count $(P$ $=0.07$ ). However, the association with symptom severity did not reach the threshold for significance after correcting for multiple testing $(P=0.0125)$. We further tested whether SNPs in the RORA gene modified the relation between cumulative trauma exposure and PTSD for diagnosis, symptom severity and symptom count. There was also no evidence of genotype-environment interaction for any PTSD phenotype ( $P$-values ranged from 0.29 to 0.97 ).

The second data set was composed of more than 2000 Iraq and Afghanistan-era US Veterans (PTSD cases and trauma-exposed controls) collected through the Mental Illness Research, Education and Clinical Center (MIRECC) housed in the Veterans Integrated Service Network 6 of the Department of Veteran Affairs. The data set included 880 European Americans (447 lifetime PTSD cases, 433 controls; 16\% female) and 1157 African Americans (586 cases, 571 controls; 32\% female). PTSD status was evaluated by the Davidson Trauma Scale ${ }^{6,7}$ and trauma exposure was measured by the Traumatic Life Events Questionnaire. ${ }^{8}$ DNA extracted from blood was genotyped by Taqman assays (Applied Biosystems) for nine SNPs in RORA (rs16942660, rs8041061, rs8042149, rs8024133, rs11071561, rs341401, rs11071587, rs11071588, rs893290). Statistical analysis was conducted using PLINK ${ }^{5}$ independently in the self-reported European-American and AfricanAmerican subjects. Under additive, recessive and dominant genetic models, controlling for the effects of gender and trauma, two SNPs were nominally associated with PTSD among African Americans under the recessive model (rs16942660, $P=0.04$; rs8041061, $P=0.03$ ), although the associations did not reach the threshold for significance after correcting for multiple testing ( $P=0.005$; Table 1$)$. Sex-stratified analyses under the dominant model identified rs341401 $(P=0.004)$ as being significantly associated with PTSD among African-American females only and rs11071587 $(P=0.008)$ as being significantly associated with PTSD among Caucasian females only. However, once this model was adjusted for the effects of resilience, ${ }^{9}$ the association was no longer significant $(P=0.037)$ after correcting for multiple testing $(P=0.005)$.

We were unable to replicate findings by Logue et al. ${ }^{1}$ or Amstadter et $a l^{2}$ of an association between variations in the RORA gene and PTSD. Our samples had high power (>99\%) to detect the effect size reported by Logue et al. ${ }^{1}$ with odds ratios between 1.8 and 2.1 under an additive model with the same risk alleles. We also found no evidence that RORA polymorphisms 